Anaesthesist 2020 $\cdot 69: 314-315$

https://doi.org/10.1007/s00101-020-00782-x

Online publiziert: 23. April 2020

(c) Springer Medizin Verlag $\mathrm{GmbH}$, ein Teil von Springer Nature 2020

\author{
J. Briegel \\ Klinik für Anaesthesiologie, LMU Klinikum, München, Deutschland
}

\title{
Virusinfektion und fehlende Beatmungsgeräte - ein Déjà-vu?
}

Im März dieses Jahres wurde aus der Lombardei und später aus New York berichtet, dass Patienten mit COVID19 und schwerem akuten respiratorischen Syndrom (SARS) verstarben, weil nicht mehr ausreichend Beatmungsgeräte verfügbar waren. Der Bundesgesundheitsminister Jens Spahn veranlasste daraufhin die Bestellung von 10.000 Beatmungsgeräten, die gesamte Jahresproduktion des größten deutschen Herstellers. In den Vereinigten Staaten wurden verschiedene Automobilbauer aufgefordert, Beatmungsgeräte herzustellen; in Italien und Spanien hatten branchenferne technische Unternehmen die Produktion einfach konstruierter Beatmungsgeräte in Kleinserie aufgenommen.

Bereits Ende März 2020 wies Stefan Dräger, Vorstandsvorsitzender der Drägerwerke AG, in einem Interview mit der Zeitschrift Der Spiegel [1] daraufhin, dass in Deutschland ein erhebliches Potenzial bei der Nutzung von Beatmungsgeräten aus dem Bereich der Rettungsmedizin und der Anästhesie liege. Er schätzte, dass hierdurch allein etwa 5000 zusätzliche Beatmungsmöglichkeiten geschaffen werden könnten. Darüber hinaus bieten verschiedene Hersteller in Deutschland und den Niederlanden Heimbeatmungsgeräte an, die bei neuromuskulären Erkrankungen oder Beatmung nach Langzeitintensivtherapie in relevanter Anzahl und sehr erfolgreich eingesetzt werden.

Zwar teilen sich die genannten Geräte zur Narkose, zum Transport im Rettungswesen oder zur Heimbeatmung einige Elemente der elektronischen Steuerung, unterscheiden sich aber konstruktiv doch erheblich von den Beatmungsgeräten der Intensivmedizin. Während Intensivrespiratoren traditionell als „offene
Systeme" mit erheblichem Frischgasverbrauch konstruiert sind, werden Narkosegeräte in der Regel als „,halbgeschlossene Systeme" mit Rückatmung und $\mathrm{CO}_{2}$ Absorption konzipiert. Dies hat erhebliche Implikationen für den klinischen Einsatz, insbesondere was Überwachung und Sicherheit im Langzeiteinsatz betrifft. Geräte für die Heimbeatmung sind primär auf assistierte Beatmungsformen ausgerichtet, häufig bei tracheotomierten Patienten mit erhaltener Spontanatmungsaktivität und unter Inkaufnahme hoher Leckagevolumina. Auch das hat für den Einsatz bei kritisch Kranken Konsequenzen, die es zu berücksichtigen gilt.

In dieser Ausgabe der Zeitschrift Der Anaesthesist stellen zwei Übersichtsarbeiten detailliert die Implikationen vor, die sich beim Einsatz von Anästhesieund Transportbeatmungsgeräten einerseits und beim Einsatz von Heimbeatmungsgeräten andererseits ergeben. Quirin Notz et al. zeigen detailliert die konstruktiven Unterschiede von Anästhesiegeräten und Transportbeatmungsgeräten auf und erläutern, in welcher Weise sie bei COVID-19-Patienten eingesetzt werden können [2]. Oliver Summ et al. stellen sehr übersichtlich und gut illustriert die in Deutschland und Österreich verfügbaren Heimrespiratoren vor und entwickeln einen Algorithmus zum ergänzenden Einsatz dieser Geräte bei einem hohen Aufkommen von beatmungspflichtigen Patienten [3]. So ergibt sich aus beiden Arbeiten, dass ein differenzierter Einsatz dieser zusätzlichen Beatmungsoptionen die verfügbaren Beatmungskapazitäten in Deutschland deutlich erhöhen kann; eine Situation, die nicht in vielen Ländern so vorhanden ist.

Man muss schon lange zurückblicken, um eine ähnliche dramatische Situation 
und ein ähnlich hohes mediales Interesse an der maschinellen Beatmung zu finden. Auch damals, in den Jahren von 1947 bis 1952 war es ein Virus, das uns gezeigt hat, welch lebensrettende Maßnahme die Beatmung von Infizierten mit bulbospinaler Poliomyelitis sein kann. Sehr schnell waren die damals verfügbaren „Eisernen Lungen“, Tankrespiratoren mit Wechseldruckbeatmung, durch erkrankte Patienten belegt, insbesondere im vom Weltkrieg gezeichneten Europa. Ausgelöst durch eine neue Welle der Poliomyelitisepidemie im Jahre 1952, entschieden sich in Kopenhagen die Anästhesisten Andersen und Ibsen, betroffene Patienten via Tracheostoma mit der damals noch unüblichen positiven Druckbeatmung zu behandeln [4]. Diese Intervention war außerordentlich erfolgreich; die Sterblichkeit sank von 87 auf $40 \%$ [5]. Dies leitete nicht nur die Ära der positiven Druckbeatmung ein, sondern auch die Entwicklung von Intensivbeatmungsgeräten mit positiver Druckbeatmung sowie die Entwicklung von Blutgasanalyse- geräten zum patientennahen Einsatz [6]. Die Intensivmedizin, wie wir sie heute kennen, nahm hier ihren Anfang.

Es darf bei all diesen technischen, medizinischen und historischen Betrachtungen jedoch nicht vergessen werden, was auch heute noch uneingeschränkt gültig ist: Der medizinische Erfolg in Kopenhagen im Jahre 1952 hing ganz entscheidend von der engagierten Behandlung und Betreuung der Erkrankten durch 250 Pflegende und 260 Medizinstudierende $\mathrm{ab}$, die in Wechselschichten für das Wohl ihrer Patienten sorgten [4].

\section{Korrespondenzadresse}

\section{Prof. Dr. J. Briegel}

Klinik für Anaesthesiologie, LMU Klinikum Marchioninistraße 15, 81377 München, Deutschland josef.briegel@med.uni-muenchen.de

Interessenkonflikt. J. Briegel gibt an, dass kein Interessenkonflikt besteht.

\section{Literatur}

1. https://www.spiegel.de/wirtschaft/unternehmen/ draegerwerk-fordert-klarheit-ueber-verteilungvon-10-000-bestellten-beatmungsgeraete-a6106fe28-bbc7-445e-96f9-671553316558

2. Notz QH, Stumpner J, Schmid B, Schlesinger T, Kredel M, Kranke P, Meybohm P, Lotz C (2020) Anästhesie- und Intensivbeatmungsgeräte - Unterschiede und Nutzbarkeit bei Covid-19 Patienten. Anaesthesist 69. https://doi.org/10. 1007/s00101-020-00781-y

3. Summ OS, Byhahn C, Kahle T, Herrmann M, Schulte C, Bergold MN, GroßM (2020) COVID-19Pandemie: Strukturierte Erweiterung von Beatmungskapazitäten mithilfe von Heimrespiratoren. Anaesthesist 69. https://doi.org/10.1007/s00101-020-00777-8

4. Andersen EW, Ibsen B (1954) The anaesthetic management of patients with poliomyelitis and respiratory paralysis. Br Med J 1:786-788

5. Lassen HC (1953) A preliminary report on the 1952 epidemic of poliomyelitis in Copenhagen with special reference to the treatment of acute respiratory insufficiency. Lancet 1:37-41

6. Astrup P, Gotzche H, Neukirch F (1954) Laboratory investigations during treatment of patients with poliomyelitis and respiratory paralysis. Br Med J 1:780-786

Hier steht eine Anzeige. 